\title{
Editorial: ASE 2009 conference trip report
}

\author{
Robert J. Hall
}

Received: 29 November 2009 / Accepted: 30 November 2009 / Published online: 9 December 2009

(C) Springer Science+Business Media, LLC 2009

In addition to introducing this issue's articles, I will report on my recent trip to the ASE Conference. My purpose in the latter is not to systematically survey the content; instead, I hope to convey one person's (subjective) experience with the latest cutting edge of the field. In this way, I hope to provide some temporal balance in Journal content with the substantial and archival (if a bit delayed compared to the conference) articles that make up the main substance of the Journal's content. Please write to me at Bob.ASEJ@gmail.com if you have an opinion pro or con about this piece.

\section{This issue's articles}

The contributions in this issue span a wide range of areas from test automation through software process and combinatorial optimization at the requirements level.

- In "Test Input Reduction for Result Inspection to Facilitate Fault Localization", Hao, Xie, Zhang, Wang, Sun, and Mei describe techniques for reducing the number of test inputs one must manually inspect when using high-coverage test suites for localizing faults in a program.

- In "Estimating the feasibility of transition paths in Extended Finite State Machines", Derderian, Hierons, Harman, and Guo describe a code-path fitness function that heuristically estimates how easy it is to randomly generate a test input that traverses a path. In this way, one can more easily construct covering sets.

- Kou, Johnson, and Erdogmus address the interesting issue of determining whether a software organization is actually following a given defined process and measuring quantitatively how close they come to doing so. In "Operational Definition and

R.J. Hall (更)

AT\&T Labs Research, Florham Park, NJ 07932, USA

e-mail: Bob.ASEJ@gmail.com 
Automated Inference of Test-Driven Development with Zorro", they explain their Zorro tool that infers this information.

- Finally, in "Finding Robust Solutions in Requirements Models", Gay, Menzies, Jalali, Mundy, Gilkerson, Feather, and Kiper discuss how the KEYS2 search algorithm can be applied not only to rapidly finding good sets of mitigations in complex requirements models (consisting of goals, risks, and mitigations), but also to characterizing the robustness of solutions using decision ordering diagrams.

\section{Trip report: ASE 2009 conference}

The Twenty-fourth IEEE/ACM International Conference on Automated Software Engineering (ASE 2009) was held 16-20 November at the Langham Hotel, Auckland, New Zealand. The General Chair, Professor John Grundy of the University of Auckland, and his team orchestrated an excellent installment of the annual conference. The venue was quite comfortable, even a bit luxurious; e.g., there were "ice caves" (?) in the hotel's spa. The schedule included long and short paper sessions, interesting keynote talks, workshops, tutorials, and the Doctoral Symposium. Finally, Auckland's temperate late spring weather encouraged attendees to take in the many natural sights around the area, such as the Rangitoto nature preserve. Accessible by ferry, Rangitoto is an island formed 600 years ago by the eruption of the eponymous volcano in the middle of the harbor. It features interesting geology, vegetation, birds, and many miles of hiking.

The technical program was dominated by two primary software engineering application areas: defect detection, including two sessions with that title, two titled "verification", and one on "testing", and maintenance and evolution, including two with that title and one on "variability". However, the conference also continued in its tradition of breadth as well as depth, with papers touching on many other areas of software engineering, including requirements, human-computer interaction, computer-supported cooperative work, automation in documentation, configuration and deployment, and software process modeling and decision support.

I'm sure it will not come as a surprise that the two dominant solution approaches were modeling and simulation in various forms, with many approaches using both of these. Of course, there are many styles and instances of both, from architectural and requirements level modeling to large scale concrete simulations to model checking (both symbolic and concrete).

While it is beyond the scope of this editorial to comprehensively summarize the whole conference, here are a few interesting ideas and questions I ran across.

- The conference's final keynote talk was given by Matt Aitken of Weta Digital Ltd. Weta Digital is a digital visual effects company, based in New Zealand, noted for having produced the digital effects for the recent Lord of the Rings trilogy and a recent King Kong remake, among others. The talk was both entertaining and technically interesting. The most interesting thing I learned was that while Weta engineers develop lots of complex modeling, simulation, and artificial-life code for, e.g., animating complex crowd scenes, ultimately the test of correctness is whether it looks good to the director. When I asked the speaker what software tools they had 
to help verify and validate all that code, he said basically very little and they could possibly use some research in that area. The deep question lurking here extends into many other areas where results are obtained from complex simulations: how do the end users know whether the simulation is producing the intended results? In this domain, I would think that there could in general be a significant risk of one of the millions of movie-goers noticing something unintended in the released animation that the director missed and drawing attention to it in an online forum. (I'm not saying this has happened yet, of course.) Many other domains depend upon the correctness of massive scale modeling and simulation, including weather and climate prediction, aerospace engineering, military systems, chip design, and even scientific research. It looks to me like a new grand challenge question for the ASE Community could be to contribute new tools and techniques in the area of $\mathrm{V} \& \mathrm{~V}$ for massive scale modeling and simulation.

- Symbolic execution continues to be a hot topic, as many papers discussed techniques using it in various ways. Of course, symbolic execution has been around since the 1970s, and combined symbolic/concrete around since the late 1980s, but this year there were several interesting and novel uses of these concepts in various papers. A couple of papers use it to reason about looping, which is a relatively little-explored use. One uses symbolic execution to summarize the effects of a loop ("Loopfrog" paper by Kroening et al.) and another uses it to judge at run time whether a loop is infinite or not ("Looper" paper by Burnim et al.). The paper by Yu et al. uses symbolic execution both forwards (from inputs to target site) to determine the possibility of a problem and then backwards (from target site to inputs) to generate a string pattern that can be used to guard against potentially problematic inputs.

- The first keynote speech, by Gerti Kappel of the Technical University of Vienna, pointed out that there is so much modeling being done in all areas of software engineering, as well as so many diverse types of modeling, that managing models has become a research challenge. She gave two specific examples, model versioning and model exchange, which are currently in a chaotic state needing new research contributions. A particularly interesting part of her talk outlined a large subset of the space of different modeling languages and their evolutionary relationships.

- Another interesting topic area that was new this year is quantum computing and its relationships to software engineering. Since practical quantum computers do not exist yet, this work depends critically on modeling and simulation and, in particular, on an advance in such to evaluate the novel search algorithm. An interesting potential challenge question that came up in the discussion was whether ASE-style program synthesis techniques (such as those invented in the 80s and 90s by Manna, Waldinger, Smith, Lowry et al.) could be applied to the problem of discovering new quantum computing algorithms, particularly those achieving the elusive exponential speedup, which are currently very few and (apparently) difficult to discover.

- Another technique appearing in the work of multiple groups is the idea of using combinatorial search algorithms to optimize parameter settings in complex models of software related phenomena. Such algorithms are familiar from artificial intelligence research, but new ones are being invented as well. In the Green et al. paper, combinatorial search is used to support software process decisions based on the 
business needs to be fulfilled, while agile and traditional processes are compared using model-search techniques in the Lemon et al. paper. And Aleti et al. apply something called ant colony optimization to the problem of deploying software.

- The research community continues its trend toward practical relevance and, in particular, languages used by practitioners today. There were papers on tools for ANSI C, Java, PHP, and Ruby on Rails, among others.

Finally, the ASE Steering Committee met, as it always does, at the annual conference to carry out its chartered duties. Among other significant decisions reached, the 2012 ASE Conference will be held in Essen, Germany, with General Chair Prof. Dr. Michael Goedicke of the University of Duisberg-Essen. Thus, the next three conferences will be held as follows. ASE 2010 - Antwerp, Belgium (September 2010); ASE 2011-Lawrence, Kansas, USA (November 2011); and ASE 2012-Essen, Germany (September 2012). 